\title{
CORRELAÇÃO ENTRE MICROSCOPIA CONFOCAL RAMAN E DISTRIBUIÇÃO DE TAMANHO DE PARTÍCULAS POR DIFRAÇÃO A LASER NA CARACTERIZAÇÃO DE MEDICAMENTOS INALATÓRIOS
}

\author{
Vinícius A. O. Delben ${ }^{12^{*}}$, Denise F. S. Petri ${ }^{{ }^{*}}$ \\ 1 -Brainfarma Indústria Química e Farmacêutica S/A., Barueri, SP \\ 2 -Instituto de Química, Universidade de São Paulo (USP), São Paulo, SP \\ viniciusdelben@gmail.com
}

\begin{abstract}
Resumo: Medicamentos inalatórios são formulações compostas por um carreador, normalmente lactose, e insumos farmacêuticos ativos (IFAs), em sua maioria broncodilatadores ou corticoides ou ambos, sendo que os IFAs representam uma fração mínima em sua composição. Além das lactoses e IFAs, as cápsulas e os dispositivos inalatórios complementam o produto e também impactam no desempenho do produto. A dispersão dos IFAs na superfície das partículas de lactose é essencial para o melhor desempenho do produto, fazendo com que o processo de mistura, bem como o tamanho de partículas dos IFAs e das lactoses sejam críticos. Um medicamento inalatório referência foi caracterizado pelas técnicas de microscopia Raman confocal e distribuição de tamanho de partículas por difração a laser (PSD). Os resultados de ambas as técnicas foram correlacionados e comparados com PSDs de IFAs de 3 diferentes fabricantes, visando identificar os pontos críticos do processo produto de medicamentos inalatórios e selecionar o melhor fabricante para o desenvolvimento de um medicamento genérico ou similar.
\end{abstract}

Palavras-chave: microscopia Raman confocal, distribuição de tamanho de partículas, medicamentos inalatórios, IFA

\section{Correlation between Raman confocal microscopy and particle size distribution by laser diffraction on the characterization of orally inhaled drug products}

\begin{abstract}
Orally inhaled drugs are formulations composed of a carrier, usually lactose, and active pharmaceutical ingredients (API), mostly bronchodilator or corticosteroids, or both, with API representing a minimal fraction in their composition. Besides lactose and API, capsules and inhalation devices complement the product and impact in product performance. The dispersion of API on the surface of lactose particles is essential for the best performance of the product, making the mixing process, as well as the particle size of API and lactose, critical. A reference inhaled drug product was characterized by confocal Raman microscopy (CRM) and laser diffraction. The results of both techniques were correlated and the particle size distribution (PSD) of API of 3 different manufacturers were compared, aiming at identifying the critical points of the inhaled drug products processing and selecting the best manufacturer for the development of a generic or similar drug product.
\end{abstract}

Keywords: Raman confocal microscopy, particle size distribution, inhalable drug products, API.

\section{Introdução}

Medicamentos inalatórios são responsáveis pela entrega local ou sistêmica dos insumos farmacêuticos ativos (IFAs) pela via pulmonar. $\mathrm{O}$ uso de medicamentos inalatórios tem grandes benefícios para o paciente, como efeito mais rápido da medicação e uso de doses menores dos IFAs. $\mathrm{O}$ uso da terapia inalatória é principalmente destinado para doenças do trato respiratório, como asma, doença pulmonar obstrutiva crônica (DPOC), fibrose cística e infecções pulmonares, todavia, a entrega sistêmica dos medicamentos pode ser usada no tratamento de diabetes, câncer e doenças neurológicas. ${ }^{1-3}$

O tamanho das partículas dos IFAs presentes em medicamentos inalatórios é bastante específico, com distribuição de tamanho de partículas (PSD - Particle Size Distribution) com a maior proporção do volume de partículas entre $1 \mu \mathrm{m}$ e $5 \mu \mathrm{m}$. Partículas abaixo de $1 \mu \mathrm{m}$ sofrem difusão e são expelidas no processo de expiração, enquanto parcelas acima de $5 \mu \mathrm{m}$ são impactadas na faringe 
e são deglutidas. ${ }^{3}$ O principal processo de obtenção de partículas dentro desta faixa é a micronização (jet-milling), em que as partículas sofrem colisão entre si através de alto vácuo. Entretanto, o material adquire muita energia estática e se aglomera, tornando crítico o processo de mistura dos IFAs com o carreador. ${ }^{1,4,5}$.

A lactose monoidratada é o carreador mais comum nos medicamentos inalatórios, por sua ótima estabilidade físico-química, baixa toxicidade, baixa higroscopicidade, apresenta grandes superfícies com capacidade de adesão das partículas e podem facilmente ser selecionadas com diferentes tamanhos de partícula e, consequentemente, diferentes propriedades reológicas. Lactoses com tamanhos menores podem melhorar a dispersão de partículas menores na mistura, porém, aumentam a coesão do pó, enquanto partículas maiores acomodam os IFAs em suas superfícies e aumentam a fluidez do pó. 6,7

A técnica de análise de distribuição de tamanho de partículas por difração a laser é um processo de dispersão de materiais sob o movimento de um fluido, normalmente água ou ar. O fluido em questão direciona as partículas através de um feixe de luz e um arranjo de detectores. Com a incidência da luz nas partículas, ocorrem fenômenos ópticos como absorção, reflexão, refração e difração. O feixe difratado nas partículas chega aos detectores, que por meio de um software, calculam o tamanho das partículas. ${ }^{8} \mathrm{O}$ PSD na indústria farmacêutica é, por via de regra, expressado em porcentagem de volume. ${ }^{8,9}$ A microscopia Raman confocal (CRM - Confocal Raman Microscopy) é formada pela junção de duas técnicas muito comuns na indústria farmacêutica, a microscopia óptica e a espectroscopia Raman. A CRM é muito usada para mapeamento químico de comprimidos, tendo como principal objetivo a engenharia reversa de produtos, deformulação de tamanho de partículas, excipientes e de processos farmacêuticos. ${ }^{10-12}$

O objetivo deste trabalho é caracterizar um medicamento inalatório pelas técnicas de PSD e CRM e correlacionar qualitativamente as duas técnicas, indicando problemas na eficácia in vivo do medicamento e no processo de mistura e selecionar o melhor fabricante de um fármaco para o desenvolvimento de um produto genérico ou similar.

\section{Experimental}

\section{CRM - microscopia Raman confocal}

Para a caracterização do medicamento referência por CRM, a formulação foi extraída das cápsulas e compactada em lâmina de alumínio. Foi realizada uma montagem por microscopia de 9x6 quadros em objetiva de 20 vezes de aumento, resultando em uma área de aproximadamente $1900000 \mu \mathrm{m}^{2}$. O equipamento utilizado foi o Renishaw inVia, modelo Qontor ${ }^{\circledR}$.

Com a área preparada, os parâmetros de análise foram programados para 1 espectro Raman a cada 1 pixel, sendo que cada pixel corresponde a 1,3 $\mu \mathrm{m}$ em ambos os eixos. Dessa maneira, foram adquiridos mais de 1 milhão de espectros Raman no mapeamento do medicamento referência. Foi usado um laser no comprimento de onda de $785 \mathrm{~nm}$, potencia de $50 \%$, integração de 1 segundo e 1 acumulação espectral por pixel.

\section{PSD - distribuição de tamanho de partículas por difração a laser}

O PSD dos IFAs no medicamento referência foi avaliado em um sistema líquido usando água como diluente por não solubilizar um dos IFAs presentes na formulação. O preparo de amostra, realizado em triplicata, consiste em adicionar $1,2 \mathrm{~g}$ da formulação em $10 \mathrm{~mL}$ de água, de maneira que a 
lactose seja solubilizada, restando cerca de $10 \mathrm{mg}$ do IFA 1, que é insolúvel. O IFA 2 está em proporção de 1:20 em relação ao IFA 1 e é levemente solúvel em água, portanto somente o IFA 1 não é solubilizado. O equipamento utilizado foi um Beckman Coulter modelo LS13320.

Para efeito comparativo, foram avaliadas 3 matérias-primas de 3 fabricantes diferentes do IFA 1 estudado na formulação. As amostras foram preparadas com a adição do mesmo surfactante usado no preparo do medicamento referência diretamente em $10 \mathrm{mg}$ das matérias-primas, homogeneizando com uma espátula ou bastão de vidro. Foram adicionados $10 \mathrm{~mL}$ de água nas amostras e homogeneizadas para suspensão das partículas. As amostras foram preparadas e analisadas em triplicata, seguindo o procedimento citado anteriormente.

\section{Resultados e Discussão}

A Figura 1 apresenta o mapeamento Raman do medicamento referência, com destaque nos círculos laranjas para as partículas maiores que $5 \mu \mathrm{m}$. Os espectros referentes ao IFA 1 estão em azul e os espectros referentes ao IFA 2 estão em vermelho, enquanto a lactose está em cinza e branco. Observa-se que a lactose é o maior componente da formulação e que o IFA 1 está em maior quantidade que o IFA 2.

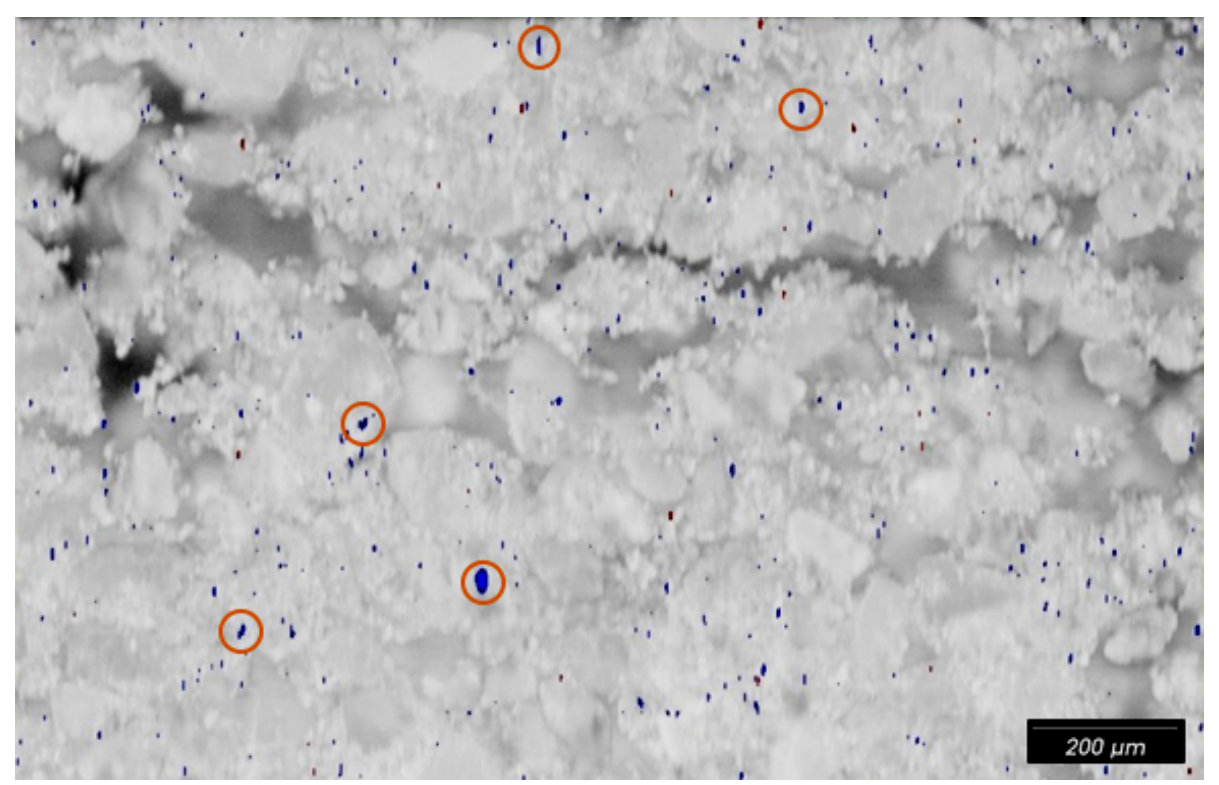

Figura 1 - Mapeamento Raman do medicamento referência com destaque (círculo laranja) para partículas maiores que $5 \mu \mathrm{m}$. Em azul, partículas do IFA 1 e em vermelho partículas do IFA 2.

As partículas maiores que $5 \mu \mathrm{m}$ correspondem à aglomerados de partículas menores que não foram dispersas no processo de mistura com a lactose na produção do medicamento. Estas partículas não produzirão o efeito farmacológico esperado do medicamento, devido aos aglomerados impactarem na faringe e serem deglutidos no uso do produto [3].

Os aglomerados além de impactarem negativamente na dose entregue do medicamento, consequentemente não geram a efetividade máxima do medicamento, sendo desperdiçado uma fração importante dos fármacos na deglutição. Um processo de mistura mais efetivo, com um 
cisalhamento maior ou maior tempo de mistura, pode desagregar essas partículas e fazer com que o medicamento seja mais efetivo no tratamento farmacológico.

Para a análise de PSD por difração a laser, o preparo das amostras é muito importante para que os resultados gerados sejam realmente condizentes com o observado na CRM. As amostras não foram colocadas em ultrassom para que os aglomerados permaneçam intactos durante a análise e não sejam quebrados. Os resultados de PSD referentes às partículas dos IFAs suspensas com o surfactante são apresentados na Figura 2 e Tabela 1, juntamente com as distribuições de tamanho de partículas dos 3 fabricantes dos IFA 1.

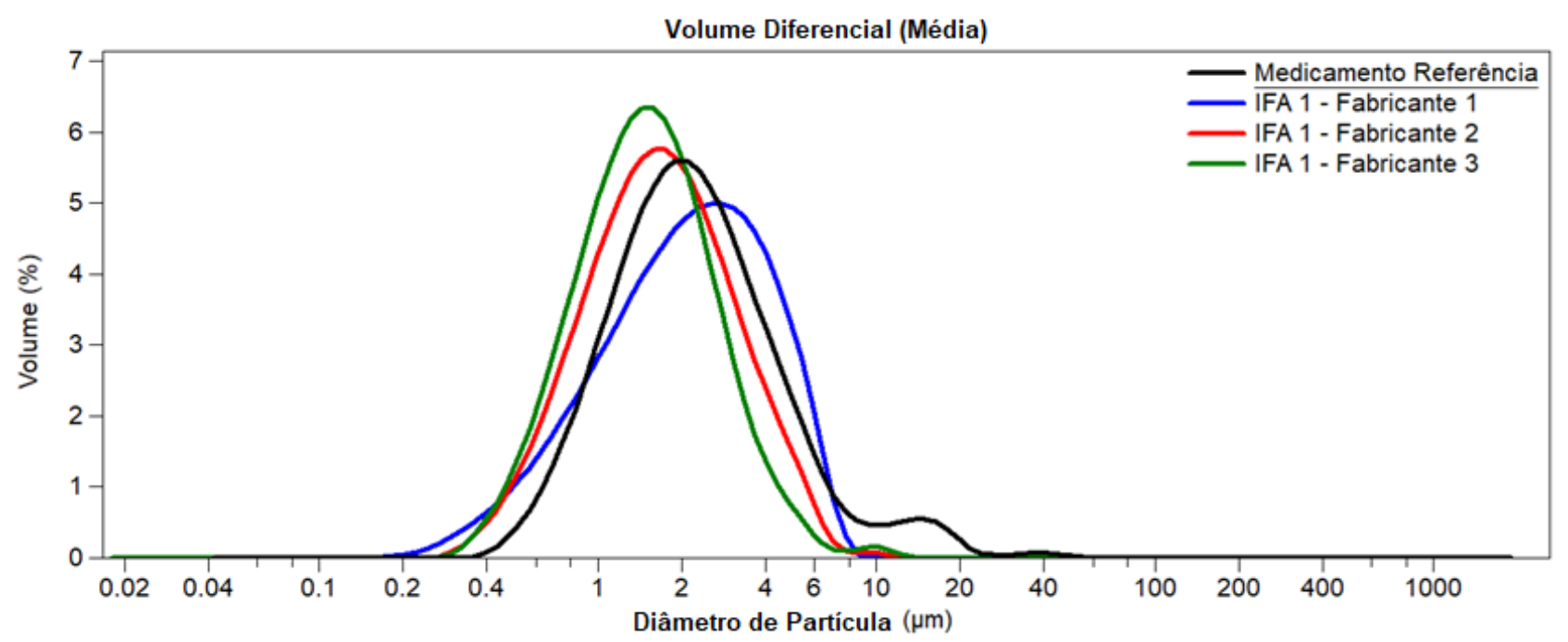

Figura 2 - Distribuição de tamanho de partículas do medicamento referência e dos fabricantes 1,2 e 3 do IFA 1.

Tabela 1 - Resultados de distribuição de tamanho de partículas do medicamento referência e dos fabricantes 1,2 e 3 do IFA 1.

\begin{tabular}{l|c|c|c|c}
\hline Amostra & Moda $(\boldsymbol{\mu m})$ & $\mathbf{d}_{\mathbf{1 0}}(\boldsymbol{\mu m})$ & $\mathbf{d}_{\mathbf{5 0}}(\boldsymbol{\mu m})$ & $\mathbf{d}_{\mathbf{9 0}}(\boldsymbol{\mu m})$ \\
\hline Medicamento Referência & 1,92 & 0,95 & 2,13 & 5,52 \\
\hline IFA 1 - Fabricante 1 & 2,79 & 0,74 & 2,15 & 4,81 \\
\hline IFA 1 - Fabricante 2 & 1,75 & 0,73 & 1,66 & 3,75 \\
\hline IFA 1 - Fabricante 3 & 1,45 & 0,69 & 1,48 & 3,10 \\
\hline
\end{tabular}

Observa-se que no histograma de PSD do medicamento referência há uma segunda moda entre 10 $\mu \mathrm{m}$ e $20 \mu \mathrm{m}$, referente aos aglomerados das partículas do IFA 1, em maior quantidade no medicamento referência e também observados no mapeamento Raman do medicamento referência.

Os PSDs do IFA 1 dos três fabricantes apresentam diferenças entre si, principalmente em relação à moda dos histogramas. $\mathrm{O}$ fabricante 2 possui a moda mais próxima do medicamento referência, entretanto, os parâmetros $\mathrm{d}_{10}, \mathrm{~d}_{50}$ e $\mathrm{d}_{90}$ é mais próximo do fabricante 1 , devido ao PSD do medicamento referência estar deslocado pelas partículas aglomeradas, entre $10 \mu \mathrm{m}$ e $20 \mu \mathrm{m}$.

Para uma melhor correlação entre os fabricantes, foi avaliada a porcentagem de volume de partículas entre $1 \mu \mathrm{m}$ e $5 \mu \mathrm{m}$, faixa de tamanho em que as partículas são inaladas. Os resultados estão na Tabela 2. 
Tabela 2 - Porcentagem em volume de partículas entre $1 \mu \mathrm{m}$ e $5 \mu \mathrm{m}$ do medicamento referência e dos três fabricantes do IFA 1.

\begin{tabular}{l|c}
\hline Amostra & \% Partículas 1 $\boldsymbol{\mu m}$ a 5 $\boldsymbol{\mu m}$ \\
\hline Medicamento Referência & 78,2 \\
\hline IFA 1 - Fabricante 1 & 75,9 \\
\hline IFA 1 - Fabricante 2 & 77,3 \\
\hline IFA 1 - Fabricante 3 & 75,1 \\
\hline
\end{tabular}

A porcentagem em volume de partículas entre $1 \mu \mathrm{m}$ e $5 \mu \mathrm{m}$ do medicamento referência é mais próxima do fabricante 2 , apesar dos parâmetros $d_{10}, d_{50}$ e $d_{90}$ não se serem os mais próximos dos três fabricantes.

\section{Conclusões}

Os resultados obtidos pela microscopia Raman confocal e distribuição de tamanho de partículas por difração a laser possibilitaram observar uma correlação qualitativa entre as técnicas na detecção de aglomerados, sendo que o processo de mistura com baixo cisalhamento é o principal responsável por não desaglomerar as partículas. Pela técnica de PSD também foi possível identificar o melhor fabricante do IFA 1 para o desenvolvimento de um medicamento genérico ou similar pela proporção de partículas na faixa de $1 \mu \mathrm{m}$ a $5 \mu \mathrm{m}$, intervalo de tamanho, onde as partículas têm atividade pulmonar.

\section{Agradecimentos}

Agradecemos ao apoio da Brainfarma e do Instituto de Química - USP.

\section{Referências}

1. Aguiar, R. et al.; Rev Port Imunoalergologia 2017, 25, 9-26.

2. Alagusundaram, M. et al.; Int. J.Res. Pharm. Sci. 2010, 1, 34-42.

3. Telko, M. J.; Hickey, A. J.; Respiratory care 2005, 50, 1209-1227.

4. Guenete, E. et al.; Int. J. Pharm 2009, 380, 80-88.

5. https://aerosol-soc.com/abstracts/dpi-inhalation-performance-optimization-api-micronizationdesign/, acessada Outubro de 2020.

6. Grasmeijer, F. et al.; PloS One 2014, 9, e87825.

7. Dickhoff, B.H. et al.; Eur J Pharm Biopharm. 2005, 59, 197-205.

8. Allen, T.; Particle size measurement, $5^{\text {th }}$ edition, v1,Chapman \& Hall: London, 1997.

9. Shekunov, B. Y.; Pharm. Res. 2007, 24, 203-227.

10. Gordon, K. C.; McGoverin, C. M.; Int. J. Pharm 2011, 417, 151-162.

11. Paudel, A.et al.; Adv.Drug Del. Rev. 2015, 89, 3-20.

12. Clarke, F. C. et al.; Anal. Chem. 2001, 73, 2213-2220. 